\title{
Performance Prediction of Solar Adsorption Refrigeration System by Ann
}

\author{
V. Baiju and C. Muraleedharan \\ Department of Mechanical Engineering, National Institute of Technology Calicut, Kerala 673601, India \\ Correspondence should be addressed to V. Baiju, baij84@yahoo.co.in
}

Received 27 January 2012; Accepted 14 February 2012

Academic Editors: I. I. El-Sharkawy and P. Trens

Copyright ( $) 2012$ V. Baiju and C. Muraleedharan. This is an open access article distributed under the Creative Commons Attribution License, which permits unrestricted use, distribution, and reproduction in any medium, provided the original work is properly cited.

\begin{abstract}
This paper proposes a new approach for the performance analysis of a single-stage solar adsorption refrigeration system with activated carbon-R134a as working pair. Use of artificial neural network has been proposed to determine the performance parameters of the system, namely, coefficient of performance, specific cooling power, adsorbent bed (thermal compressor) discharge temperature, and solar cooling coefficient of performance. The ANN used in the performance prediction was made in MATLAB (version 7.8) environment using neural network tool box.In this study the temperature, pressure, and solar insolation are used in input layer. The back propagation algorithm with three different variants namely Scaled conjugate gradient, Pola-Ribiere conjugate gradient, and Levenberg-Marquardt (LM) and logistic sigmoid transfer function were used, so that the best approach could be found. After training, it was found that LM algorithm with 9 neurons is most suitable for modeling solar adsorption refrigeration system. The ANN predictions of performance parameters agree well with experimental values with $R^{2}$ values close to 1 and maximum percentage of error less than 5\%. The RMS and covariance values are also found to be within the acceptable limits.
\end{abstract}

\section{Introduction}

The conventional refrigeration systems require mechanical energy as the driving source and are responsible for the emission of $\mathrm{CO}_{2}$ and the other greenhouse gases such as CFCs and HFCs which are considered major cause for ozone layer depletion. From this context, the adsorption refrigeration system attains a considerable attention in 1970s due to the energy crisis and ecological problems related to the use of CFCs and HFCs. Research has proved that the adsorption refrigeration technology has a promising potential for competing with the conventional vapour compression refrigeration systems. In comparison with the vapour compression refrigeration systems, adsorption refrigeration systems have the benefits of energy savings if powered by waste heat or solar energy, like simpler control, absence of vibration, and low operation cost.

The major attraction of solar adsorption refrigeration is that its working fluids satisfy the Montreal protocol on ozone layer depletion and the Kyoto protocol on global warming
[1]. The consumption of low grade energy by the adsorption units does not possess any problems of emission of greenhouse gases. Furthermore, solar-power-based refrigerator is simple and is adaptable for small, medium, or large systems [2]. By use of ozone-friendly refrigerants and ability to utilize the renewable energy sources, the adsorption systems can be preferred as an alternative to the conventional refrigeration systems $[3,4]$. The low COP and SCP values as compared to the conventional refrigeration systems are the barriers for the commercialization of the adsorption refrigeration systems $[5,6]$. For the improvement of the system, a detailed computational and thermodynamic analysis must be carried out.

The thermodynamic analyses of adsorption systems are complex because of the complex differential equations involved. Instead of solving complex differential equations and applying the limited number of experimental data, faster and simpler solutions can be obtained by using artificial neural network. ANNs are able to learn the key information patterns within multidimensional information domain. The 


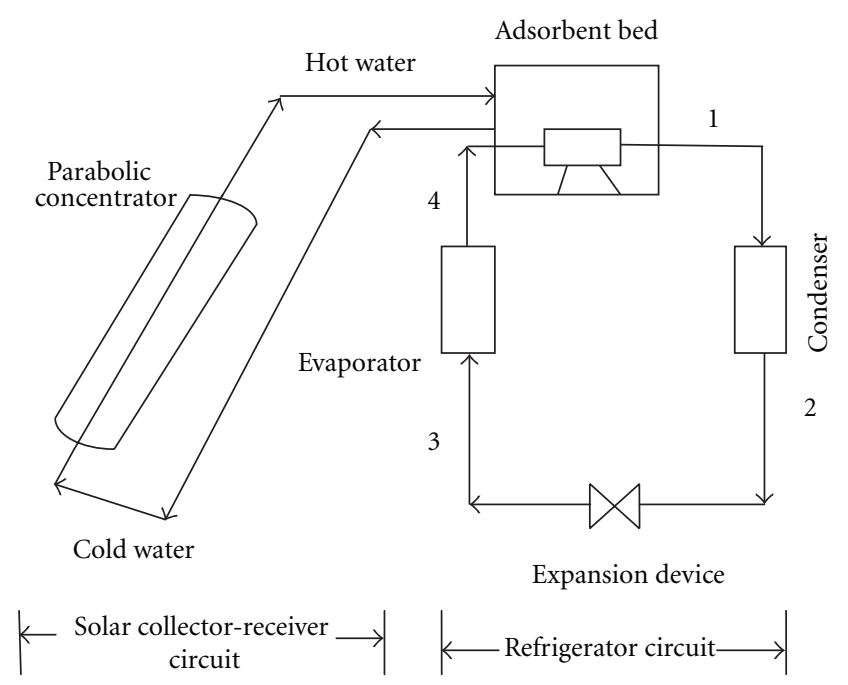

FIgURE 1: Schematic of solar adsorption refrigeration system.

use of ANN for the performance prediction and simulation of complex system is increasingly becoming popular in the last few years.

The application of artificial neural network for the exergy performance prediction of solar adsorption refrigeration system working at different conditions is necessary nowadays for making the analysis simple. Many earlier studies have reported the application of artificial neural network for the performance predictions of vapour compression refrigeration systems such as for direct expansion heat pump [7], for modeling solar cooling systems [8], and for modeling cascade refrigeration systems [9] with the acceptable accuracy. Recently some works about the use of ANN in energy systems have been published [10-17].

From the brief literature review cited, it can be observed that many investigators have used the artificial neural network for the performance prediction of various thermal energy systems. No much work has reported the applicability of artificial neural network for the performance prediction of the solar adsorption refrigeration system. Hence, in the present work, the ANN approach is used for investigating the performance of solar adsorption refrigeration system. Utilising the data obtained from the experimental system, an ANN model for the system is developed. With the use of this model, various performance parameters of the system, namely, the coefficient of performance, specific cooling power, thermal compressor discharge temperature, and solar cooling coefficient of performance are predicted and compared with the actual values.

\section{Description of the System and Experimental Data}

2.1. Experimental Setup. The solar-assisted adsorption refrigeration system consists of a parabolic solar concentrator, water tank, adsorbent bed, condenser, expansion device (capillary tube), and evaporator as shown in Figure 1, and its photograph is shown in Figure 2. The specifications of

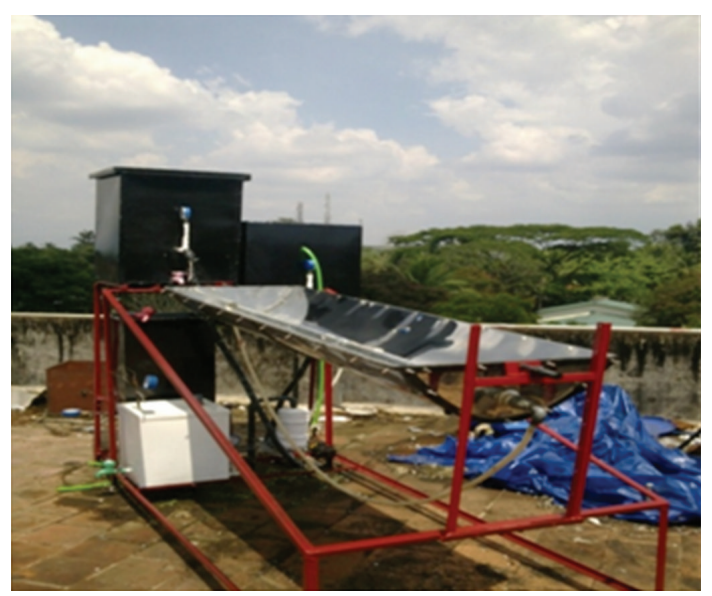

FIgUre 2: Photograph of the experimental setup.

TABLE 1: The specifications of main components of solar adsorption refrigeration system.

\begin{tabular}{ll}
\hline Component & Technical specification \\
\hline Condenser & Capacity: $200 \mathrm{~W}$ \\
& Water cooled \\
Evaporator & Material: copper \\
& Capacity: $150 \mathrm{~W}$ \\
Expansion device & Capillary tube \\
Adsorbent bed & Material: stainless steel \\
Parabolic solar concentrator & Solar concentrator of area $3 \mathrm{~m}^{2}$ \\
& made of stainless steel \\
& Activated carbon \\
Adsorbent & Type: granular particle size: \\
& 0.25 mm \\
Adsorbate & Mass: $1.5 \mathrm{~kg}$ \\
& R134 a
\end{tabular}

the components used in the system are given in Table 1. The experimental set up is located in the Solar Energy Laboratory at National Institute of Technology Calicut, Kerala, India. The solar adsorption refrigeration system is tested under the meteorological conditions of Calicut (latitude of $11.15^{\circ} \mathrm{N}$, longitude of $75.49^{\circ} \mathrm{E}$ ) during April 2011.

2.2. Experimentation. Water gets heated starting from morning while flowing through the solar concentrator by natural circulation. When the hot water is circulated around the adsorbent bed, the temperature in the adsorbent bed increases. This causes the vapour pressure of the adsorbed refrigerant to reach up to the condensing pressure. The desorbed vapour is liquefied in the condenser. The high pressure liquid refrigerant is expanded through the expansion device to the evaporator pressure. The low pressure liquid refrigerant then enters the evaporator where it evaporates by absorbing the latent heat of evaporation. In the evening, the hot water from the tank should be drained off and is refilled with cold water. The temperature of the adsorbent bed reduces rapidly and the pressure in the adsorber drops below the evaporator pressure. The experiments are carried 
out keeping the evaporator temperature constant. The same procedure is repeated for the different evaporator loads.

2.3. Measurements. A digital pyranometer with an accuracy of $\pm 5 \mathrm{~W} / \mathrm{m}^{2}$ is placed near the solar collector to measure the instantaneous solar insolation. Pressure is measured during heating (desorption) of refrigerant, that is, condensing pressure and during cooling (adsorption), that is, evaporator pressure. The pressure gauges are fixed at the adsorbent bed in order to measure the pressure inside the adsorbent bed at each stage of adsorption and desorption processes. The temperature at various points in the solar adsorption refrigeration system is measured by calibrated Ttype (Copper-Constantan) thermocouples. The various temperatures observed are (1) temperature of the adsorbent bed during various processes, (2) temperature of the refrigerant at inlet and outlet of the condenser, expansion device exit, and evaporator outlet, (3) temperature of water entering the water tank, and (4) temperature of chilled water in the evaporator box.

\section{Performance Parameters}

The main performance parameters used for the present study are cycle coefficient of performance, specific cooling power, and solar cooling coefficient of performance [18].

3.1. Cycle COP. Cycle COP is defined as the ratio of cooling effect to the total energy required for desired cooling effect:

$$
\mathrm{COP}=\frac{\text { cooling effect }}{\text { total energy input }}=\frac{Q_{e}}{Q_{T}} .
$$

The total energy input to the system is given by.

$$
Q_{T}=Q_{\text {isosteric heating }}+Q_{\text {desorption }} \text {. }
$$

The total heat supplied to the system is equal to the enthalpy change of solar heated water

$$
Q_{T}=\dot{m} c_{p}\left(T_{f i}-T_{f o}\right) .
$$

Cooling effect is as follows:

$$
Q_{e}=m_{w} C_{P w}\left(\Delta T_{w}\right) .
$$

3.2. Specific Cooling Power (SCP). Specific cooling power indicates the size of the system as it measures the cooling output per unit mass of adsorbent per unit time. Higher SCP values indicate the compactness of the system:

$$
\begin{aligned}
\text { SCP } & =\frac{\text { Cooling effect }}{\text { Cycle time per unit of adsorbent mass }} \\
& =\frac{Q_{e}}{m_{a} \times \tau_{\text {cycle }}} .
\end{aligned}
$$

3.3. Solar COP. Since the system is solar-powered, the solar coefficient of performance is also to be defined. This is
TABLE 2: Uncertainty in different parameters.

\begin{tabular}{lc}
\hline Description & Total uncertainty (\%) \\
\hline COP $_{\text {cycle }}$ & $\pm 6.04 \%$ \\
Heat input to the system & $\pm 1.09 \%$ \\
Solar cooling COP & $\pm 3.12 \%$ \\
Specific cooling power & $\pm 2.91 \%$ \\
\hline
\end{tabular}

defined as the ratio of cooling effect to the net solar energy input:

$$
\text { Solar COP }=\frac{Q_{e}}{Q_{s}}
$$

\section{Uncertainty Analysis}

Uncertainties in the experiments can arise from the selection, condition, and calibration of the instruments, environment, observation, and test planning. A more precise method of estimating uncertainty has been presented by Holman [19]. The method is based on a careful specification of uncertainties in the various primary experimental measurements. These measurements are then used to calculate some desired results of the experiments. In the present study, the pressure, temperatures, and solar insolation were measured by using the instruments. The total uncertainties of the various calculated parameters are shown in Table 2 and its calculation procedure is given in the appendices.

The total uncertainty arising due to independent variables is given by

$$
w_{R}=\left[\left(\frac{\partial R}{\partial x_{1}} w_{1}\right)^{2}+\left(\frac{\partial R}{\partial x_{2}} w_{2}\right)^{2}+\cdots+\left(\frac{\partial R}{\partial x_{n}} w_{n}\right)^{2}\right]^{1 / 2}
$$

The result $R$ is a given function in terms of independent variables. Let $w_{R}$ be the uncertainty in the result and let $w_{1}, w_{2}, \ldots, w_{n}$ be the uncertainties in the independent variables.

\section{Neural Network Design}

Artificial intelligent (AI) systems are widely used as a technology offering an alternative way to tackle complex and ill-defined problems. They can learn from examples in the sense that they are able to handle noisy and incomplete data, are able to deal with the nonlinear problems, and once trained, can perform prediction and generalization at high speed [12]. Artificial neural network system resembles human brain in two aspects the knowledge is acquired by the network through learning process, and the neuron connection strengths known as synaptic weights are used to store the knowledge. Artificial neural network is an interconnected assembly of simple processing elements, units, or nodes, whose functionality is loosely based on the animal neuron. The fundamental processing element of a neural network is a neuron. Basically a biological neuron receives input information from other sources, combines them in other ways, performs generally a nonlinear operation, and outputs 


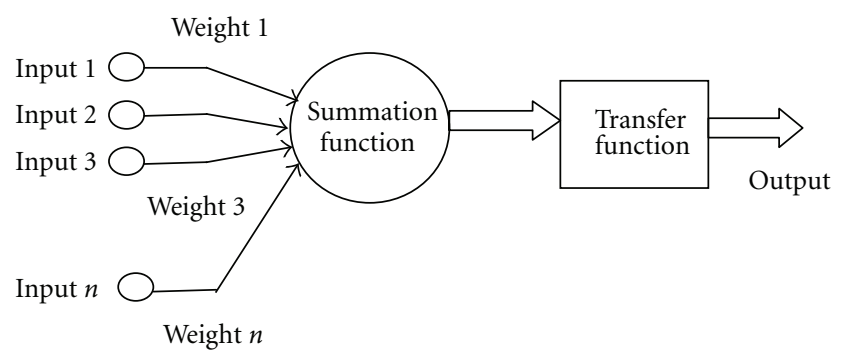

Figure 3: Artificial neuron.

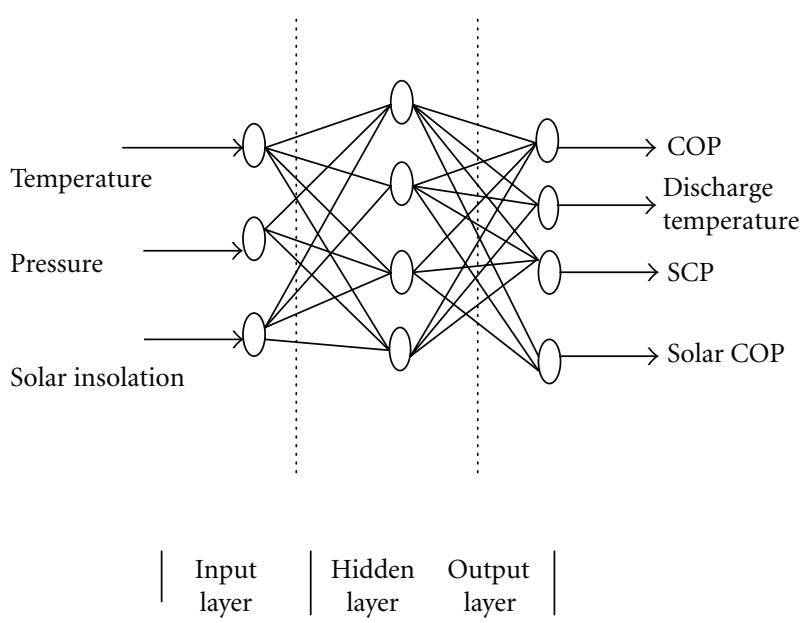

FIGURE 4: Neural network for the performance prediction of solar adsorption refrigeration system.

the final results. The network usually consists of an input layer, some hidden layer, and an output layer. An artificial neuron is shown in Figure 3.

An important stage of a neural network is the training step, in which an input is introduced in the network together with the desired output, and the weights are adjusted so that the network attempts to produce the desired output. There are different learning algorithms. A popular algorithm is a standard backpropagation algorithm which has different variants. It is very difficult to know which algorithm is faster for the given problem. The artificial neural network with backpropagation algorithm learns by changing the weights, and these changes are stored as knowledge. Some statistical methods, in fact root mean square (RMS), correlation coefficient $\left(R^{2}\right)$, covariance (COV), and mean absolute percentage error (MAPE), are used for comparison [14]:

$$
\begin{aligned}
\mathrm{RMS} & =\left(\frac{1}{n} \sum_{j=1}^{n}\left[t_{j}-o_{j}\right]^{2}\right)^{1 / 2}, \\
R^{2} & =1-\left(\frac{\sum_{j}\left[t_{j}-o_{j}\right]^{2}}{\sum_{j}\left(o_{j}\right)^{2}}\right), \\
\mathrm{COV} & =\frac{\mathrm{RMS}}{\sum_{j} o_{j}} * 100, \\
\mathrm{MAPE} & =\frac{o-t}{o} * 100 .
\end{aligned}
$$

\section{Modelling of Solar Adsorption Refrigeration System by Artificial Neural Network}

The performance parameters such as cycle COP, SCP, discharge temperature, and solar $\mathrm{COP}$ are predicted by using artificial neural network. The architecture of an ANN used for the performance prediction of SAR indicating input and output is shown in Figure 4.

In this study, the pressure, temperature, and solar intensity are used as input parameters whereas the cycle coefficient of performance, specific cooling power, discharge temperature, and solar cooling coefficient of performance are predicted in the output layer. The back propagation algorithm is used in feed forward single hidden layer network. The variants of the algorithm used in the study are Scaled Conjugate Gradient (SCG), Pola-Ribiere Conjugate Gradient (CGP), and Levenberg-Marquardt (LM). The inputs and outputs are normalized in the range $0-1$. Logistic sigmoid (log sig) transfer function is being used in ANN.

The transfer function used is given by

$$
f(Z)=\frac{1}{1+e^{-Z}}
$$

where $z$ is the weighted sum of inputs.

The artificial neural network used in SAR modeling was made in the MATLAB (version 7.8) environment using 
TABLE 3: Statistical values of the different networks evaluated.

\begin{tabular}{lccc}
\hline Algorithm & $R^{2}$ & RMS & COV \\
\hline LM-8 & 0.9985 & 0.0204 & 0.308 \\
LM-9 & 0.99987 & 0.01035 & 0.158 \\
LM-10 & 0.9878 & 0.01078 & 0.159 \\
SCG-8 & 0.9874 & 0.0224 & 0.876 \\
SCG-9 & 0.9544 & 0.0245 & 0.190 \\
SCG-10 & 0.9837 & 0.0359 & 0.204 \\
CGP 8 & 0.9874 & 0.0245 & 0.198 \\
CGP 9 & 0.9751 & 0.0213 & 0.163 \\
CGP 10 & 0.9832 & 0.035 & 0.83 \\
\hline
\end{tabular}

TABLE 4: Average values of performance parameters.

\begin{tabular}{lc}
\hline Parameters & Value \\
\hline Refrigerating effect $(\mathrm{W})$ & 64.4 \\
Cycle COP & 0.334 \\
Solar COP & 0.0685 \\
SCP $(\mathrm{W} / \mathrm{kg})$ & 42.5 \\
\hline
\end{tabular}

a neural network tool box. In the training, an increased number of neurons from 5 to 10 are used in hidden layer to define the output accurately. The output of network is compared with the desired output at each presentation, and errors are computed. These errors are backpropagated to the neural network for adjusting the weight such that the errors decrease with each iteration and ANN model approximates the desired output.

The available data obtained from the experimental observations are divided into training and testing sets. The data set consists of 90 input values. From these, 80 data sets are used for training and the remaining is used for testing the network. During each step, the performance of the network is studied by using the different statistical performance parameters such as $R^{2}$, RMS, and COV values. In every step, the performance of the network is tested and it is decided that the network consists of single hidden layer with 9 neurons and L-M variant is the optimum network for the particular system. The performance parameters of the network with log-sig transfer function and different variants are shown in Table 3.

\section{Results and Discussion}

Average values of performance parameters of the system obtained by conducting different experiments are shown in Table 4.

From Table 4, it is clear that the average value of solar COP is very much less than the cycle coefficient of performance. The solar COP is a parameter that is defined by considering the performance of solar concentrator. The cycle $\mathrm{COP}$ is calculated by using the total heat content of solarheated water in water tank. The maximum energy absorbed by the water in absorber of a solar concentrator is only 20$35 \%$ during peak sunshine. The remaining heat is lost due

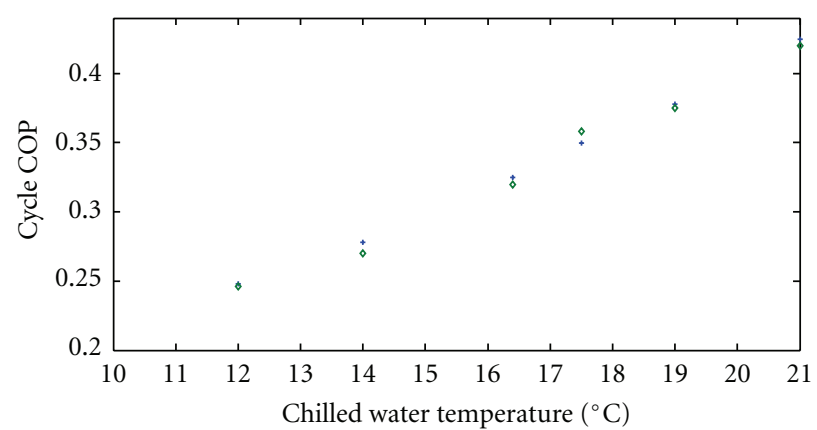

- Experimental
- ANN predicted

FIGURE 5: Comparison of actual and ANN predicted COP values.

to the high irreversibility associated with the heat transfer in solar concentrator surfaces. This leads to the very low solar coefficient of performance as compared to the cycle COP for the solar adsorption refrigeration system.

The experimental and ANN predicted results with statistical values such as RMS, COV, $R^{2}$, and MAPE for the three different parameters are shown in Table 5 .

The maximum percentage of error is $1.451 \%, 4.08 \%$, 1.17 , and $1.617 \%$ for the cycle COP, SCP discharge temperature (DT), and solar COP, respectively. The results also show that $R^{2}$ values are very close to 1 for all the data and RMS values are very small. It is clear that the neural model gives a very accurate representation of the statistical data over the full range of operating conditions and indicates good accuracy of the neural network to represent the performance predictions of the solar adsorption refrigeration system. As seen from the results, the performance parameters are obviously calculated within the acceptable uncertainties.

The experimental and ANN predicted values of cycle $\mathrm{COP}$, specific cooling power, discharge temperature, and solar cooling coefficient of performance are shown in Figures $5-8$.

Figure 5 shows the ANN predicted results and experimentally calculated values of cycle COP with different evaporator loads. In this case an ANN predicted value yields a correlation coefficient of 0.99872 . The RMS and COV values are found to be 0.00364 and 0.00182 , respectively. The coefficient of performance is an important parameter considered for the rating of SAR system, which is calculated based on the evaporator capacity and heat input to the adsorbent bed from solar-heated water.

A plot of experimental and ANN predicted values for the specific cooling power against chilled water temperature is shown in Figure 6. These predictions yield a correlation coefficient of 0.99503 RMS and COV values of 0.9678 and 0.003417 , respectively. The comparison shows that the ANN predicted values are closer to experimental results. Specific cooling power is also an important parameter that determines the size of the system.

Figure 7 shows the experimentally measured and ANN predicted values of solar coefficient of performance for different evaporator loads. The ANN predictions for the solar 
TABLE 5: Comparison between the experimental and ANN predicted results.

\begin{tabular}{lccccccccccc}
\hline & \multicolumn{3}{c}{ Experimental } & \multicolumn{4}{c}{ ANN predicted } & \multicolumn{4}{c}{$\%$ error } \\
Cycle COP & SCP & Solar COP & DT & Cycle COP & SCP & Solar COP & DT & Cycle COP & SCP & Solar COP & DT \\
\hline 0.248 & 37 & 0.045 & 58 & 0.246 & 37.5 & 0.0448 & 58.4 & 0.80 & -1.35 & 0.44 & -0.69 \\
0.275 & 42 & 0.055 & 60.4 & 0.271 & 42.8 & 0.0558 & 60.8 & 1.451 & -1.90 & -0.148 & -0.65 \\
0.325 & 45 & 0.062 & 61.82 & 0.328 & 45.8 & 0.061 & 61 & -0.92 & -1.77 & 1.617 & 1.42 \\
0.35 & 49 & 0.072 & 63.25 & 0.354 & 51 & 0.073 & 64 & -1.15 & -4.08 & -1.388 & -1.175 \\
0.378 & 52 & 0.082 & 64.3 & 0.375 & 51.8 & 0.0826 & 64.5 & 0.798 & 0.384 & -0.72 & -0.305 \\
0.425 & 58 & 0.084 & 65.86 & 0.42 & 58.2 & 0.0849 & 65.8 & 1.176 & -0.344 & -1.07 & 0.091 \\
\hline
\end{tabular}

$R^{2}: 0.99872,0.99503,0.99905,0.9814$.

RMS: $0.003642,0.9678,0.0008,0.5167$.

COV: $0.00182,0.003417,0.002,0.00148$.

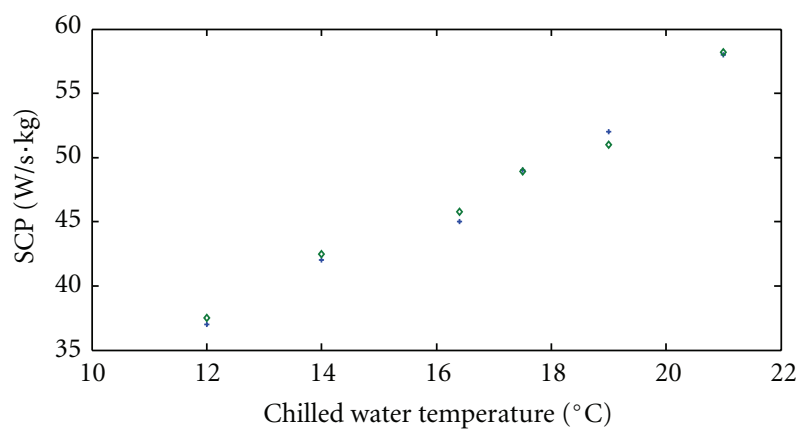

+ Experimental

- ANN predicted

FIGURE 6: Comparison of experimental and ANN predicted values of specific cooling power.

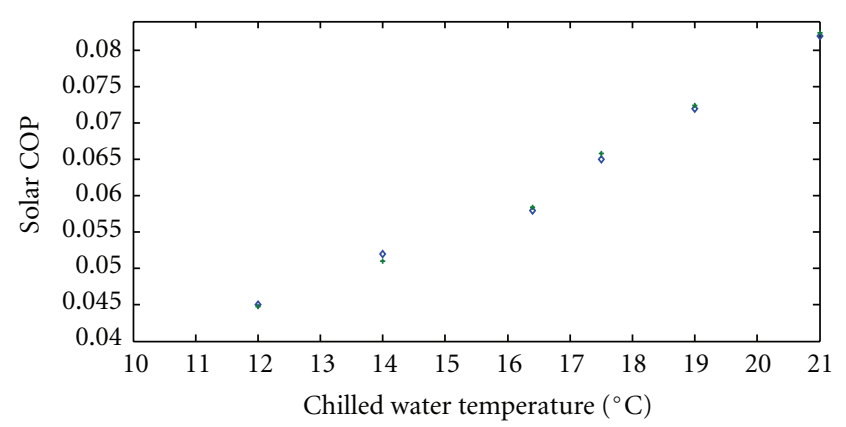

- Experimental

- ANN predicted

FIGURE 7: Comparison of ANN predicted and experimental values of solar cooling COP.

COP yield a correlation coefficient of 0.99905 with RMS and covariance values of 0.0008 and 0.002 , respectively. The results confirmed that ANN predicts exactly the solar cooling COP for different chilled water temperature.

The ANN predicted and experimentally measured values of the thermal compressor discharge temperature are depicted in Figure 8. From the figure it is clear that the ANN predictions are in well agreement with the experimental

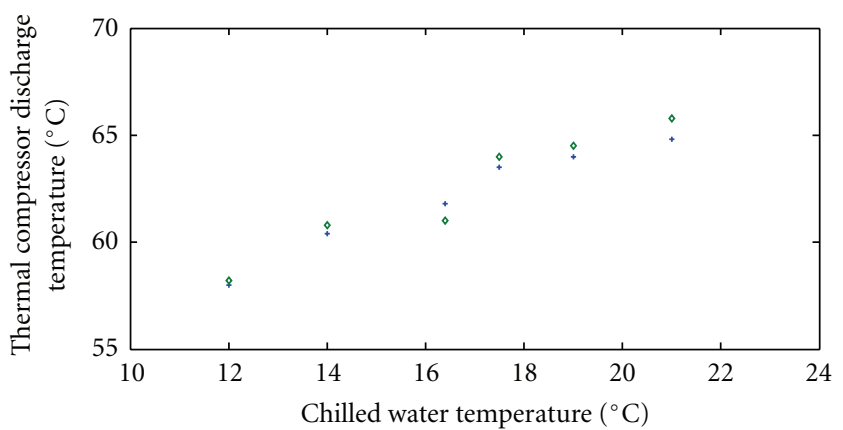

- Experimental

- ANN predicted

FIGURE 8: Comparison of ANN predicted and experimentally measured compressor discharge temperature.

results of discharge temperature. The comparison gives a correlation coefficient of 0.9814 , and the RMS and COV values are found to be 0.5167 and 0.00148 , respectively.

\section{Conclusions}

The artificial neural network approach has been applied to the solar hybrid adsorption refrigeration system as an alternative to the classical approaches, which are usually complicated, require an enormous amount of the experimental data, and may yield inaccurate results. In order to gather the data for training and testing the proposed ANN, an experimental system has been set up and tested for its performance at different evaporator loads. Using the three different parameters, namely, temperature, pressure, and solar insolation an ANN model based on the back propagation algorithm was proposed, The performance of ANN predictions was measured using the three criteria root mean square error, correlation coefficient, and coefficient of variance. The network model demonstrated good results with correlation coefficients in the range of 0.98146-0.99905 and percentage of error $0.344 \%-4.08 \%$. This study reveals that, with the use of neural network, solar adsorption refrigeration systems can be modeled with a high degree of accuracy. 


\section{Appendices}

A.

The total uncertainty arising due to independent variables is given by

$$
w_{R}=\left[\left(\frac{\partial R}{\partial x_{1}} w_{1}\right)^{2}+\left(\frac{\partial R}{\partial x_{2}} w_{2}\right)^{2}+\cdots+\left(\frac{\partial R}{\partial x_{n}} w_{n}\right)^{2}\right]^{1 / 2} .
$$

The result $R$ is a given function in terms of independent variables. Let $w_{R}$ be the uncertainty in the result and let $w_{1}, w_{2}, \ldots, w_{n}$ be the uncertainties in the independent variables. COP of the system is a function of several variables each subject to an uncertainty

$$
\begin{aligned}
\mathrm{COP}_{\text {sys }} & =f\left(\dot{m}, h, Q_{g}\right), \\
\mathrm{COP}_{\text {sys }} & =\frac{Q_{\text {evap }}}{Q_{g}}=0.196, \\
\frac{\partial \mathrm{COP}}{\partial Q_{\text {evap }}} & =\frac{1}{Q_{g}}, \\
\frac{\partial \mathrm{COP}}{\partial Q_{g}} & =-\frac{Q_{\text {evap }}}{Q_{g}^{2}}, \\
w_{R} & =\left[\left(\frac{1}{Q_{g}} w_{g}\right)^{2}+\left(\frac{Q_{\text {evap }}}{Q_{g}^{2}} w_{\text {evap }}\right)^{2}\right]^{1 / 2} \\
& =0.01184=6.04 \% .
\end{aligned}
$$

\section{B. Levenberg Marquardt Back Propagation}

LM is a network training function that updates weight and bias values according to Levenberg-Marquardt optimization. It is the fastest backpropagation algorithm in the toolbox, and is highly recommended as a first-choice supervised algorithm, although it does require more memory than other algorithms.

LM algorithm takes these inputs: initial training record created, training data, validation data, test data, and it returns the trained network. Training occurs according to LM training parameters, shown here with their default values.

Maximum number of epochs to train 100.

100: $\quad$ Maximum number of epochs to train

0: $\quad$ Performance goal

5: $\quad$ Maximum validation failures

1: $\quad$ Factor to use for memory/speed tradeoff

$1 e-10$ : Minimum performance gradient

0.001: Initial mu

0.1 : mu decrease factor

10: $\quad$ mu increase factor

1e10: Maximum mu

25: $\quad$ Epochs between displays (NaN for no displays)

0: $\quad$ Generate command-line output

1: $\quad$ Show training GUI

Inf: Maximum time to train in seconds.
Validation vectors are used to stop training early if the network performance on the validation vectors fails to improve or remains the same for max_fail epochs in a row. Test vectors are used as a further check that the network is generalizing well, but do not have any effect on training.

LM can train any network as long as its weight, net input, and transfer functions have derivative functions.

Backpropagation is used to calculate the Jacobian $(j X)$ of performance (perf) with respect to the weight and bias variables $(X)$. Each variable is adjusted according to Levenberg-Marquardt:

(i) $j j=j X^{*} j X$,

(ii) $j e=j X^{*} E$,

(iii) $d X=-\left(j j+I^{*} m u\right) \backslash j e$, where $E$ is all errors and $I$ is the identity matrix.

The adaptive value mu is increased by mu_inc until the change above results in a reduced performance value. The change is then made to the network and mu is decreased by mu_dec.

The parameter mem_reduc indicates how to use memory and speed to calculate the Jacobian $j X$. If mem_reduc is 1 , then trainlm runs the fastest, but can require a lot of memory. Increasing mem_reduc to 2 cuts some of the memory required by a factor of two, but slows trainlm somewhat. Higher states continue to decrease the amount of memory needed and increase training times.

Training stops when any of the following conditions occurs.

(i) The maximum number of epochs (repetitions) is reached.

(ii) The maximum amount of time is exceeded.

(iii) Performance is minimized to the goal.

(iv) The performance gradient falls below min_grad.

(v) mu exceeds mu_max.

(vi) Validation performance has increased more than max_fail times since the last time it decreased (when using validation).

\section{Nomenclature}

$\mathrm{C}_{p}: \quad$ Specific heat $(\mathrm{kJ} / \mathrm{kg} \mathrm{K})$

COP: Coefficient of performance

SCP: Specific cooling power

$\dot{m}: \quad$ Mass flow rate $(\mathrm{kg} / \mathrm{s})$

$\mathrm{p}: \quad$ Pressure $(\mathrm{Pa})$

T: $\quad$ Temperature $(\mathrm{K})$

Q: $\quad$ Rate of heat transfer $(\mathrm{kW})$

E: Exergy

S: Entropy

h: $\quad$ Enthalpy $(\mathrm{kW})$

$\dot{W}$ : $\quad$ Rate of work transfer $(\mathrm{kW})$. 


\section{Subscripts}

$\begin{array}{ll}\text { ads: } & \text { Adsorption/adsorbent bed } \\ \text { des: } & \text { Desorption } \\ \text { c: } & \text { Cooling } \\ \text { cyc: } & \text { Cycle } \\ \text { i/in; } & \text { Inlet } \\ \text { o/out: } & \text { Outlet } \\ \text { f: } & \text { Fluid } \\ \text { a: } & \text { Adsorbent } \\ \text { r: } & \text { Refrigerant } \\ \text { s: } & \text { Solar. }\end{array}$

\section{References}

[1] E. E. Anyanwu, "Environmental pollution: restructuring the refrigeration industry as a way out," Environment Protection Engineering, vol. 26, no. 4, pp. 17-27, 2000.

[2] E. E. Anyanwu, "Review of solid adsorption solar refrigerator I: an overview of the refrigeration cycle," Energy Conversion and Management, vol. 44, no. 2, pp. 301-312, 2003.

[3] D. S. Jung and R. Radermacher, "Performance simulation of single-evaporator domestic refrigerators charged with pure and mixed refrigerants," International Journal of Refrigeration, vol. 14, no. 4, pp. 223-232, 1991.

[4] Y. S. Chang, M. S. Kim, and S. T. Ro, "Performance and heat transfer characteristics of hydrocarbon refrigerants in a heat pump system," International Journal of Refrigeration, vol. 23, no. 3, pp. 232-242, 2000.

[5] K. Sumathy, K. H. Yeung, and L. Yong, "Technology development in the solar adsorption refrigeration systems," Progress in Energy and Combustion Science, vol. 29, no. 4, pp. 301-327, 2003.

[6] N. M. Khattab, "A novel solar-powered adsorption refrigeration module," Applied Thermal Engineering, vol. 24, no. 17-18, pp. 2747-2760, 2004.

[7] M. Mohanraj, S. Jayaraj, and C. Muraleedharan, "Modeling of a direct expansion solar assisted heat pump using artificial neural networks," International Journal of Green Energy, vol. 5, no. 6, pp. 520-532, 2008.

[8] M. Mohanraj, S. Jayaraj, and C. Muraleedharan, "Exergy analysis of direct expansion solar-assisted heat pumps using artificial neural networks," International Journal of Energy Research, vol. 33, no. 11, pp. 1005-1020, 2009.

[9] Y. H. Kuang, K. Sumathy, and R. Z. Wang, "Study on a directexpansion solar-assisted heat pump water heating system," International Journal of Energy Research, vol. 27, no. 5, pp. 531$548,2003$.

[10] M. Hosoz and H. M. Ertunc, "Modelling of a cascade refrigeration system using artificial neural network," International Journal of Energy Research, vol. 30, no. 14, pp. 1200-1215, 2006.

[11] S. A. Kalogirou and M. Bojic, "Artificial neural networks for the prediction of the energy consumption of a passive solar building," Energy, vol. 25, no. 5, pp. 479-491, 2000.

[12] A. Pacheco-Vega, M. Sen, K. T. Yang, and R. L. McClain, "Neural network analysis of fin-tube refrigerating heat exchanger with limited experimental data," International Journal of Heat and Mass Transfer, vol. 44, no. 4, pp. 763-770, 2001.

[13] A. Chouai, S. Laugier, and D. Richon, "Modeling of thermodynamic properties using neural networks: application to refrigerants," Fluid Phase Equilibria, vol. 199, no. 1-2, pp. 5362, 2002.
[14] A. Palau, E. Velo, and L. Puigjaner, "Use of neural networks and expert systems to control a gas/solid sorption chilling machine," International Journal of Refrigeration, vol. 22, no. 1, pp. 59-66, 1999.

[15] H. Bechtler, M. W. Browne, P. K. Bansal, and V. Kecman, "New approach to dynamic modelling of vapour-compression liquid chillers: artificial neural networks," Applied Thermal Engineering, vol. 21, no. 9, pp. 941-953, 2001.

[16] S. A. Kalogirou, S. Panteliou, and A. Dentsoras, "Artificial neural networks used for the performance prediction of a thermosiphon solar water heater," Renewable Energy, vol. 18, no. 1, pp. 87-99, 1999.

[17] T. T. Chow, G. Q. Zhang, Z. Lin, and C. L. Song, "Global optimization of absorption chiller system by genetic algorithm and neural network," Energy and Buildings, vol. 34, no. 1, pp. 103-109, 2002.

[18] K. Sumathy and L. Zhongfu, "Experiments with solarpowered adsorption ice-maker," Renewable Energy, vol. 16, no. 1-4, pp. 704-707, 1999.

[19] J. P. Holman, Experimental Methods for Engineers, Tata McGraw-Hill, New Delhi, India, 7th edition, 2007. 

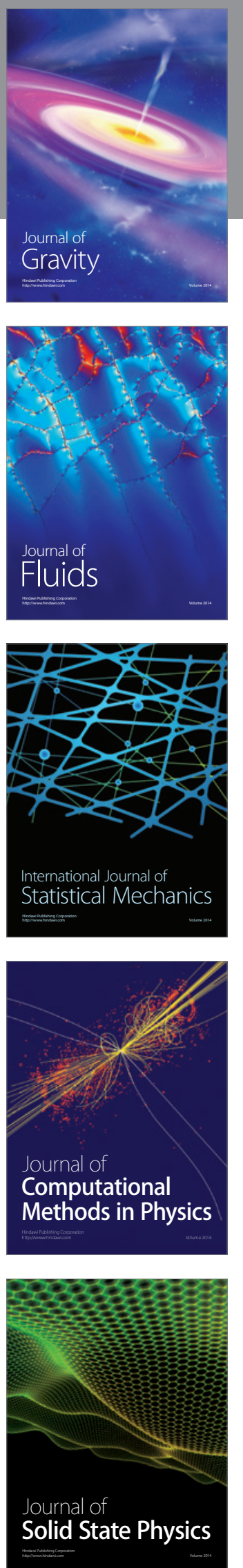
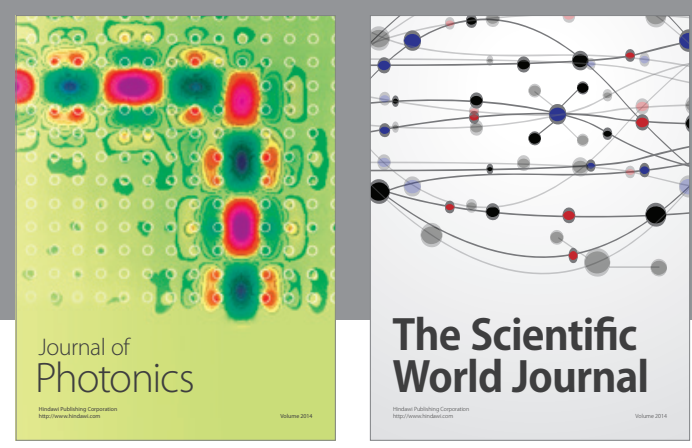

The Scientific World Journal

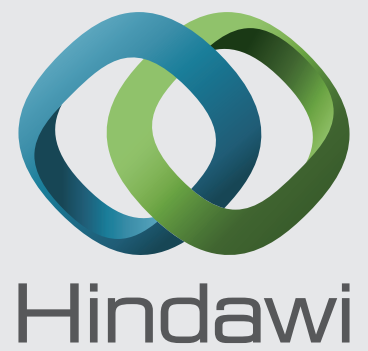

Submit your manuscripts at http://www.hindawi.com
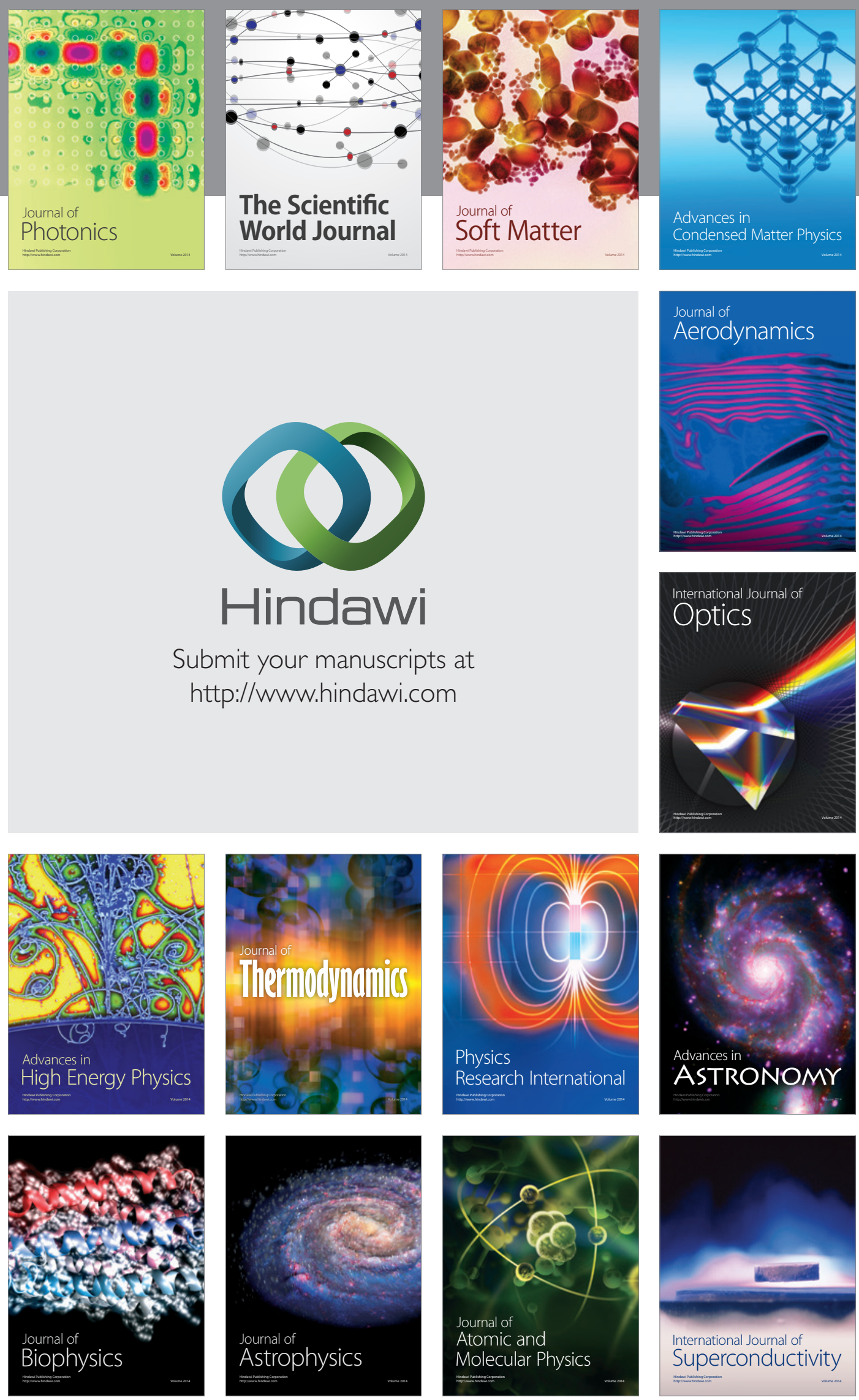
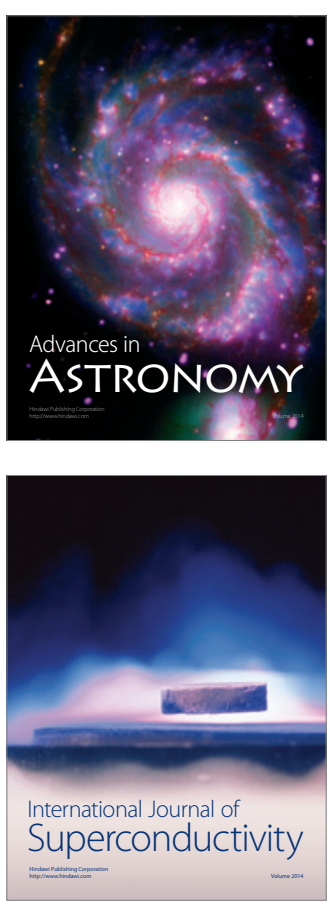